\title{
EDITORIAL
}

\section{Electronic Cigarette: A Savior or a Lure?}

\author{
${ }^{1}$ Kamran H Awan, ${ }^{2}$ R Hegde
}

How to cite this article: Awan $\mathrm{KH}$, Hegde R. Electronic Cigarette: A Savior or a Lure? World J Dent 2017;8(3):155-156.

Source of support: Nil

\section{Conflict of interest: None}

Are electronic cigarettes (e-cigarettes) the lesser of the two evils compared with smoking or just a newer, shinier health threat? To anyone who has followed the controversy around the perceived merits and health risks of e-cigarettes, the research and reactions can seem confusing. Unfortunately, there is no long-term safety data about e-cigarettes and the information we have suggests that e-cigarettes have a complex mix of potential harms and benefits.

The savior: E-cigarettes are almost certainly less harmful than conventional cigarettes. Conventional cigarette smoke consists of toxic gases, such as carbon monoxide and hydrogen cyanide. Conventional cigarette smoke also contains an ultrafine suspension of gummy residue, known as tar. Most of the carcinogens related to conventional cigarette smoke are found in the tar. E-cigarettes do not produce the tar or the toxic gases found in conventional cigarette smoke. An e-cigarette contains a cartridge of fluid, popularly known as e-liquid. E-liquid is made up of nicotine and flavorings dissolved in propylene glycol and glycerol. The e-liquid is superheated by a battery-powered vaporizer, converting it into a mist, i.e., inhaled, or "vaped."

Studies about e-cigarettes and smoking behavior show conflicting results. E-cigarettes were mildly helpful in kicking the habit in one clinical trial. ${ }^{1}$ In other studies, e-cigarette use did not increase quit rates, ${ }^{2}$ or was even associated with a higher risk of continuing to smoke. ${ }^{3} \mathrm{~A}$ recent review concluded that real-world use of e-cigarettes is associated with lower quit rates. ${ }^{4}$

The lure: E-cigarettes, like conventional cigarettes, also contain nicotine that may have several negative health effects. Nicotine is highly addictive and may lead to changes in the brain that increase the risk of addiction

\footnotetext{
${ }^{1,2}$ College of Dental Medicine, Roseman University of Health Sciences, South Jordan, Utah 84095, USA

Corresponding Author: Kamran H. Awan, College of Dental Medicine, Roseman University of Health Sciences, 10894 S. River Front Parkway, South Jordan, Utah 84095, USA, Phone: +18018781099, e-mail: kamranhabibawan@gmail.com
}

to other drugs, especially in young people. Nicotine may also impair prefrontal brain development in adolescents, leading to attention deficit disorder and poor impulse control. Chronic nicotine exposure may lead to insulin resistance and type II diabetes, although this risk may be offset by the well-known appetite-suppressant effects of nicotine. Inhaled nicotine increases heart rate and blood pressure. These potential harms of nicotine in e-cigarettes are particularly worrisome in view of increasing rates of its use in teenagers. ${ }^{5}$

The e-liquid used in e-cigarettes may also be a household hazard. Many e-liquids have candy and fruit flavoring and packaging that make them attractive to children. Children exposed to e-cigarette devices and nicotine liquid are $>2.5$ times more likely to have a severe outcome than children exposed to cigarettes. In recent years, cases of nicotine poisoning from e-liquid have skyrocketed, with accidental ingestions of e-liquid by kids rising by $1,500 \%{ }^{6}$

Flavored e-cigarettes may pose another health threat. They often contain a chemical compound called diacetyl, which is associated with a rare lung disease called bronchiolitis obliterans that causes permanent damage to the bronchioles (the tiniest airways in the lungs). Propylene glycol and glycerol, the major components of e-liquids, are not thought to be dangerous on their own. However, they may decompose when heated by the vaporizer, and be transformed into toxic compounds, such as formaldehyde, a very carcinogenic compound. This is more common with newer vaporizers that use high wattages.

Verdict: While not harmless, e-cigarettes have demonstrated a much more favorable toxicological profile than conventional cigarettes. Their viability as a public health strategy to end the use of conventional cigarettes will likely be determined by their ability to decrease smokers' exposure to levels of harmful constituents as well as their ability to deliver nicotine efficiently and serve as a sufficient replacement for smoking.

\section{REFERENCES}

1. Bullen C, Howe C, Laugesen M, McRobbie H, Parag V, Williman J, Walker N. Electronic cigarettes for smoking cessation: a randomised controlled trial. Lancet 2013 Nov 16;382(9905):1629-1637.

2. Grana RA, Popova L, Ling PM. A longitudinal analysis of electronic cigarette use and smoking cessation. JAMA Intern Med 2014 May;174(5):812-813. 
3. Al-Delaimy WK, Myers MG, Leas EC, Strong DR, Hofstetter CR. E-cigarette use in the past and quitting behavior in the future: a population-based study. Am J Public Health 2015 Jun;105(6):1213-1219.

4. Kalkhoran S, Glantz SA. E-cigarettes and smoking cessation in real-world and clinical settings: a systematic review and meta-analysis. Lancet Respir Med 2016 Feb;4(2):116-128.
5. Awan KH. Experimentation and correlates of electronic nicotine delivery system (electronic cigarettes) among university students - a cross sectional study. Saudi Dent J 2016 Apr;28(2):91-95.

6. Kamboj A, Spiller HA, Casavant MJ, Chounthirath T, Smith GA. Pediatric exposure to e-cigarettes, nicotine, and tobacco products in the United States. Pediatrics 2016;137(6).pii:e20160041. 